\title{
Plant WEE1 Kinase Interacts with a 14-3-3 Protein, GF14 $\omega$ but a Mutation of WEE1 at S485 Alters Their Spatial Interaction
}

\author{
Anne Lentz Grønlund ${ }^{1,2,3}$, J. Richard Dickinson ${ }^{1}$, Peter Kille ${ }^{1}$, John L. Harwood ${ }^{1}$, Robert J. Herbert ${ }^{2}$, \\ Dennis Francis ${ }^{1, *}$ and Hilary J. Rogers ${ }^{1}$ \\ ${ }^{1}$ School of Biosciences, Cardiff University, Park Place, Cardiff. CF10 3TL, UK \\ ${ }^{2}$ Institute of Science and the Environment, University of Worcester, Henwick Grove, Worcester, WR2 6AJ UK \\ ${ }^{3}$ Rothamsted Research, Harpenden AL5 2JQ, UK
}

\begin{abstract}
In animals, 14-3-3 proteins bind two cell cycle proteins WEE1 and CDC25 stabilising their phosphorylated state. We report here for the first time interactions between WEE1 and 14-3-3 proteins both in vitro and in vivo in plants. The Arabidopsis 14-3-3 family partitions into either an Epsilon or Non-Epsilon group. In a yeast 2-hybrid screen Arabidopsis WEE1 interacted with the Non-Epsilon group. Subsequently, we focussed on Non-Epsilon GF14 $\omega$, a 14-3-3 expressed more strongly in proliferative than in non-proliferative cells and which is able to rescue a cell cycle checkpoint mutant in yeast. The WEE1/GF14 $\omega$ interaction was confirmed by an in vitro co-immunoprecipitation assay and in vivo in tobacco BY-2 cells by bi-molecular fluorescence complementation. Sub-cellular interaction between WEE1 and GF14 $\omega$ occurs in the nucleus of interphase cells. Additionally an interaction was very occasionally observed in the cross wall between cells. Their small stature and independent observations of callose, of the type that typically forms in new cell plates, suggests that this additional interaction might be occurring at cytokinesis. An S485A mutation of WEE1 abolished this interaction in vitro and altered the spatial interaction in vivo indicating that this is a likely regulatory phosphorylation target for the WEE1/GF14 $\omega$ interaction.
\end{abstract}

Key Words: 14-3-3 Proteins, Arabidopsis thaliana, bi-molecular fluorescence complementation cell cycle, WEE1.

\section{INTRODUCTION}

In eukaryotes the cell cycle is regulated by cyclin dependent kinases (CDKs) at G1/S and G2/M [1] in a mechanism conserved from yeast to animals [2]. CDKs are tightly regulated by their association with cyclins through phosphoregulation. At the G2/M transition, CDKs are also phosphoregulated positively by CDC25 phosphatase [3], and negatively by WEE1 kinase, which has been identified in yeasts [4], animals [5] and plants [6-8]. In normal animal cell cycles, WEE1 is located in the nucleus during interphase but is partitioned away from chromosomes from late prophase to early telophase [9]. Nuclear WEE1 kinase activity is high during interphase but absent during mitosis [5] whilst CDK1-cyclinB1 is located in the cytoplasm in interphase and in the nucleus at G2/M [1]. Thus spatial and molecular controls prevent unscheduled mitosis occurring in interphase and entrain the CDK to be active at the G2/M transition in mitotically competent cells. However, we know very little about spatial control of the plant cell cycle.

The Ataxia-telangiectasia mutated/related (ATM/ATR) proteins and in $S$. pombe the RAD3 protein are induced if DNA replication is perturbed or DNA is damaged [10-12].

*Address correspondence to this author at the School of Biosciences, Cardiff University, Park Place, Cardiff. CF10 3TL, UK; Tel: + 44-29-20875086; Fax: +44- 29- 2087- 4305; E-mail: francisd@ @ardiff.ac.uk
They trigger a pathway of DNA dependent kinases that repair the DNA defect and phosphorylate and activate the checkpoint kinases, CHK1 and/or CHK2. These kinases participate in the stabilisation of WEE1 kinase activity ensuring that the phosphorylation of the CDK/cyclin complex is maintained and that the cell is arrested in G2 [13]. CHK1/2 also phosphorylates and inactivates CDC25 phosphatase to keep the $\mathrm{CDC} / \mathrm{CyclinB}$ complex inactive. When DNA replication is normalised or DNA damage is repaired $\mathrm{CDC} 25$ is dephosphorylated and restores the activity of the CDK/cyclin complex [14, 15].

In yeast and animal checkpoints, the phosphorylations of WEE1 and CDC25 are at least partly stabilised by the binding of 14-3-3 proteins [16-21]. These proteins have an important role in cell cycle checkpoints because they bind and protect phosphorylated sites on both WEE1 and CDC25, thereby maintaining their activation or inactivation, respectively [20, 22, 23]. 14-3-3 proteins bind their target proteins via high affinity phosphorylation-dependent binding motifs: RSXpSXP (mode I) and RXXXpSXP (mode II) [24, 25]. Seven 14-3-3s have been identified in humans and 13 in Arabidopsis [26]. Although the overall amino acid sequence of 14-3-3 proteins is highly conserved [27], the Arabidopsis $14-3-3 \mathrm{~s}$ are divided into two groups, Epsilon and NonEpsilon, based on their amino acid sequence [26]. 14-3-3 proteins are expressed in a wide range of tissues and cell types but so far only two reports suggest that plant 14-3-3s are involved in plant cell cycle regulation $[28,29]$. 
In Arabidopsis, weel T-DNA insertion lines are hypersensitive to hydroxyurea and zeocin, agents that induce the DNA replication and DNA damage checkpoints, respectively, supporting a role for WEE1 in these checkpoint mechanisms [30]. Although over-expression of WEE1 results in a $\mathrm{G} 2 / \mathrm{M}$ block, in non-stressed conditions the T-DNA insertion lines seem to develop normally raising doubts about a regulatory role for Arath;WEE1 in a normal plant cell cycle [30]. This contrasts with other eukaryotes in which WEE1 is an essential gene $[1,2,4]$. This divergent role for plant WEE1 led us to ask whether its regulation through protein-protein interaction is also different in plants.

The aim of the work presented here was thus to test the hypothesis that Arabidopsis WEE1 interacts with 14-3-3 proteins and to localise this interaction within plant cells. We demonstrated an interaction between Arath;WEE1 and GF14 $\omega$ both in vitro and in vivo and identified both the location of the interaction in vivo and a likely phosphoregulatory target for this interaction.

\section{MATERIALS AND METHODS}

\section{Constructs and Y2H Screens}

For the bait plasmid, the coding sequence of Arath; WEE1 was amplified using primers Arath;WEE1 FW 5'- CAT-GGAGAATTCATGTTCGAGAAGAACG -3' and Arath;WEE1 RV 5'- ACGTTCGACCTCAACCTCGAATCCTATC -3' and fused in-frame with the GAL4 DNAbinding domain in the pBD-GAL4-Cam vector using EcoRI and SalI sites. For the target plasmids, the coding sequences of GF14 $\omega, \lambda, \kappa, \chi, \varphi, \psi, v$ and $v$ were amplified using primers Arath;GF14 $\omega$ FW 5'- CGGGATCCATGGCGTCTGGGCGTGAA -3' and Arath;GF14 $\omega$ RV 5'- CGGAATCCTCACTGCTGTTCCTCGGTCG -3'; Arath;GF14 $\lambda$ FW 5'- CGGGATCCATGGCGGCGACATTAGGCA -3' and Arath;GF14 $\lambda$ RV 5'- CGGAATCCTCAGGCCTCGTCCATCTGCT -3'; Arath;GF14א FW 5'- CGGGATCCATGGCGACGACCTTAAGCA -3' and Arath;GF14к RV 5'- CGGAATCCTCAGGCCTCATCCATCTGCT -3'; Arath; GF14 $\psi$ FW 5'- AAGGATCCATGTCGACAAGGGAAGAGAATG -3' and Arath;GF14 $\psi$ RV 5'- AATTGCTAGCTTACTCGGCACCATCGGGCT -3'; Arath;GF14v FW 5'- AAGGATCCATGTCGTCTTCTCGGGAAGAG -3' and Arath;GF14v RV 5'- AATTGCTAGCTCACTGCCCTGTCTCAGCTG -3'; Arath;GF14v FW 5'- AAGGATCCATGTCCTCTGATTCGTCCCGG -3' and Arath;GF14v RV 5'- AATTGCTAGCTCACTGCGAAGGTGGTGGTT-3'; Arath;GF14 $\varphi$ FW 5'- AAGGATCCATGGCGGCACCACCAGCAT-3' and Arath;GF14 $\varphi$ RV 5'- AATTGCTAGCTTAGATCTCCTTCTGTTCTTCAG-3'; Arath;GF14 $\chi$ FW 5'- AAGGATCCATGGCGACACCAGGAGCTTC -3' and Arath;GF14 $\chi$ RV 5' - AATTGCTAGCCTAGGATTGTTGCTCGTCAGC -3'. The coding sequences were fused in-frame with the GAL4 DNA-binding domain of the pAD-GAL4-2.1 vector using BamHI and NheI sites. The insertion of the coding sequences into the $\mathrm{Y} 2 \mathrm{H}$ vectors was confirmed by sequencing.

Both bait and target vector were transformed into the budding yeast strain YPH499 (MATa ura3-52 lys2-801 ade2-101 trp1 463-901 his34200 leu241, gal4-542 gal80538 LYS2::UAS GAL1-TATAGAL1-HIS3 URA3::UAS GAL4 17mers(x3)-TATACYC1-lacZ (Stratagene) and plated onto trp-/leu- minimal medium containing $40 \mathrm{mM} 3$-amino1, 2, 4-triazole (3-AT; Sigma). Plates were incubated at $30^{\circ} \mathrm{C}$ and colonies were re-patched onto his minimal medium. His + patches were assayed for $\beta$-galactosidase activity using a filter lift assay (Stratagene).

\section{ONPG Assay}

The assay was performed by inoculating transformed YRG2 cells into $5 \mathrm{~mL}$ SGR medium $(1.67 \mathrm{~g} / \mathrm{L}$ Difco Yeast Nitrogen Base (w/o amino acids), $5 \mathrm{~g} / \mathrm{L}$ ammonium sulphate, $20 \mathrm{~g} / \mathrm{L}$ galactose, $10 \mathrm{~g} / \mathrm{L}$ raffinose $\pm 20 \mathrm{~g} / \mathrm{L}$ Difco bacto agar) and cultures were incubated in a Gallenkamp orbital incubator at $160 \mathrm{rpm}$ at $30^{\circ} \mathrm{C}$ until an $\mathrm{OD}_{600} 0.6-0.8$. Cells were harvested by centrifugation in an MSE Centaur 2 centrifuge for $10 \mathrm{~min}$ at $4000 \mathrm{rpm}$ and the pellet was resuspended in 5 $\mathrm{mL}$ of Z-buffer $\left(10.7 \mathrm{~g} / \mathrm{L} \quad \mathrm{Na}_{2} \mathrm{HPO}_{4} \cdot 2 \mathrm{H}_{2} \mathrm{O}, \quad 6.2 \mathrm{~g} / \mathrm{L}\right.$ $\left.\mathrm{NaH}_{2} \mathrm{PO}_{4} \cdot 2 \mathrm{H}_{2} \mathrm{O}, 0.75 \mathrm{~g} / \mathrm{L} \mathrm{KCl}, 0.246 \mathrm{~g} / \mathrm{L} \mathrm{MgSO}_{4} \cdot 7 \mathrm{H}_{2} \mathrm{O}\right)$ and placed on ice. $\mathrm{OD}_{600}$ was measured and to each sample was added one drop of $0.1 \%$ SDS and two drops of chloroform. Tubes were vortexed for $15 \mathrm{sec}$ and incubated at $30^{\circ} \mathrm{C}$ for 15 min and $160 \mu \mathrm{L}$ of $4 \mathrm{mg} / \mathrm{mL}$ ONPG (Sigma) were added. Tubes were vortexed well for $10 \mathrm{~s}$ and incubated at $30^{\circ} \mathrm{C}$ for $20 \mathrm{~min}$. After $20 \mathrm{~min}$ samples were removed and $400 \mu \mathrm{L}$ of 1 $M$ sodium carbonate were added. Samples were spun down and $\mathrm{OD}_{420}$ and $\mathrm{OD}_{550}$ of the supernatant were measured. Miller units were calculated as follows: $U=1000 \times\left[\left(\mathrm{OD}_{420}\right)\right.$ $\left.-\left(1.75 \times \mathrm{OD}_{550}\right)\right] /\left[(\right.$ time $) \times$ (volume) $\left.\times \mathrm{OD}_{600}\right]$, where time is the incubation time with ONPG ( $\mathrm{min}$ ) and the volume is the volume of initial culture used $(\mathrm{mL})$.

\section{Constructs and Co-Immunoprecipitation}

Constructs were made using the pESC-TRP yeast epitope tagging vector (Stratagene) carrying coding sequences: Arath;GF14w only, Arath;WEE1 only, Arath;GF14w/Arath; WEE1. The coding sequence of Arath;GF14w was PCRamplified using primers FW: 5'-ATGGGGATCCCAATGGCGTCTGGGCGTGAAG-3' and RV: 5'-GGATCCTCACTGCTGTTCCTCGG-3' and inserted into the BglII site of the pESC-TRP vector to introduce a FLAG epitope tag at the N-terminus. The coding sequence of Arath;WEE1 was PCR amplified using primers FW: 5'- ATCGGGATCCATGTTCGAGAAGAACGGAA-3' and RV: 5'- GCTAGTCGACACCTCGAATCCTATCAAAC-3' and inserted in the pESC-TRP vector using the BamHI and SalI sites to introduce a c-myc epitope tag at the C-terminus. Insertions of Arath;WEE1 and Arath;GF14 $\omega$ into vectors were confirmed by sequencing.

The constructs were transformed into the budding yeast strain YPH499 (ura3-52 lys2-801 ade-101ochre trp1- $\Delta 63$ his $3-\Delta 200$ leu2- $\Delta 1)$. Proteins were extracted from the yeast cells by vortexing with glass beads in lysis buffer $(50 \mathrm{mM}$ Hepes (pH 7.5), $100 \mathrm{mM} \mathrm{NaCl}, 10 \%$ glycerol, $60 \mathrm{mM} \beta$ glycerophosphate, $1 \mathrm{mM}$ sodium vanadate, $0.5 \mathrm{mM}$ phenylmethylsulfonyl fluoride (PMSF), complete protease inhibitors: 1 tablet $/ 10 \mathrm{ml}$ (Roche)). Protein extracts $(\sim 1 \mathrm{mg})$ were bound to $40 \mu \mathrm{L}$ Anti-FLAG M2 affinity gel (Sigma) in a volume of $1 \mathrm{ml}$ by rotation at $4^{\circ} \mathrm{C}$ over night. The affinity gel was subsequently washed three times with TBS and bound proteins were eluted by adding $0.1 \mathrm{M}$ glycine $\mathrm{pH}$. 3.5 to the affinity gel. Eluates were mixed with 2x SDS sample buffer [1\%SDS, 45 mM Tris- $\mathrm{HCl}(\mathrm{pH} 6.8), 50 \mathrm{mM}$ DTT, $10 \%$ glycerol, $0.1 \mathrm{mg} \mathrm{ml}^{-1}$ bromophenol blue] and separated by 
SDS-PAGE and western-blotted. Duplicate blots were probed with FLAG (1:8,000 primary antibody dilution) and c-myc (1:100 primary antibody dilution) monoclonal antibodies (Sigma) or WEE1 (1:1000 primary antibody dilution) polyclonal antibody (Sigma-Genosys).

The WEE1 antibody was raised in rabbit using the peptide DADAADGDNKDFILC located at amino acid position 87 in the N-terminal regulatory domain of tobacco (Nicotiana tabacum) WEE1, Nicta;WEE1. The peptide was synthesized chemically and checked to $>50 \%$ purity. It was conjugated to keyhole limpet haemocyanin carrier using equal amounts (w/w, 2-3 mg) of carrier and peptide for use as an antigen. Antigens were injected into the rabbits in 100-200 $\mu \mathrm{g}$ amounts, subcutaneously in rabbits at 2 -week intervals over a 70 day period, and the appearance of antibodies was determined by ELISA. The antibody specificity was tested against Nicta;WEE1 and Arath;WEE1 expressed in $E$. coli and against total protein from tobacco BY2 cells and Arabidopsis seedlings. The antibody identified a band of the correct molecular weight for WEE1 from both tobacco and Arabidopsis (data not shown). Proteins were visualised using ECL western blotting reagents (Amersham Biosciences).

\section{Constructs, Stable Transformation of Tobacco BY-2 Cell Cultures and BiFC}

BiFC vectors were kindly donated by Dr. J. Kudla, Münster University, Germany. For constructing the pSPYNE $35 \mathrm{~S}$ vectors, the coding sequences of Arath;WEE1 and Arath;GF14 $\omega$ were PCR amplified using primers WEE1 FW: 5'-ATACTAGTATGTTCGAGAAGAACGGAAG-3' and RV: 5'- TAGGTACCACCTCGAATCCTATCAAACA3'; GF14w FW: 5'-ATACTAGTATGGCGTCTGGGCGTGAAGA-3' and RV: 5'- TAGGTACCCTGCTGTTCCTCGGTCG-3'. The coding sequences were fused in-frame to the N-terminal part of the YFP using SpeI and KpnI sites. For constructing the pSPYCE $35 \mathrm{~S}$ vectors, the coding sequences of Arath;WEE1 and Arath;GF14w were PCR amplified using primers WEE1 FW: 5'- ATACTAGTATGTTCGAGAAGAACGGAAG-3' and RV: 5'- TACTCGAGACCTCGAATCCTATCAAACA-3'; GF14w FW: 5' ATACTAGTATGGCGTCTGGGCGTGAAGA-3' and RV: 5'- TACTCGAGCTGCTGTTCCTCGGTCG-3'. The coding sequences were fused in-frame to the C-terminal part of the YFP using SpeI and XhoI sites. The insertions of Arath; WEE1 and Arath;GF14 $\omega$ into the respective vectors were confirmed by sequencing.

Stable transformation of tobacco BY-2 cells was achieved by the method of An [43] modified with the addition of $20 \mu \mathrm{M}$ acetosyringon (Sigma-Aldrich) during co-cultivation of the tobacco BY-2 cells with the Agrobacterium (LBA4404) carrying the pSPYNE 35S constructs. Transformants were selected on solidified BY2 medium $(0.8 \%$ agar) supplemented with $250 \mu \mathrm{g} / \mathrm{ml}$ Timentin and 80 $\mu \mathrm{g} / \mathrm{ml}$ hygromycin. Calli were cultured in $50 \mathrm{ml} \mathrm{BY-2}$ medium, $250 \mu \mathrm{g} / \mathrm{ml}$ Timentin and $80 \mu \mathrm{g} / \mathrm{ml}$ hygromycin until stationary phase ( $1-3$ weeks).

For the BiFC experiments, the stable pSPYNE 35S lines were transiently transformed with the pSPYCE $35 \mathrm{~S}$ constructs in Agrobacterium (EHA105). Ten ml aliquots of LB medium were inoculated with a single colony of freshly streaked Agrobacterium (EHA105) carrying the pSPYCE $35 \mathrm{~S}$ vectors. Cultures were incubated overnight in a Gallenkamp orbital incubator at $160 \mathrm{rpm}$ at $30^{\circ} \mathrm{C}$. Three dayold sub-cultures of the stable tobacco BY-2 pSPYNE $35 \mathrm{~S}$ cell lines were used for the transient transformations (prior to the transient transformation, the stable tobacco BY-2 cell lines were sub-cultured without hygromycin at least twice). To $7 \mathrm{ml}$ of the stable tobacco BY-2 pSPYNE $35 \mathrm{~S}$ cell culture, $200 \mu \mathrm{M}$ acetosyringon was added. The acetosyringon-treated cells were then co-cultivated with $200 \mu \mathrm{l}$ of the overnight Agrobacterium (EHA105) carrying the pSPYCE $35 \mathrm{~S}$ constructs in Petri dishes containing BY-2 agar without selection. Plates were wrapped in Nesco film and incubated in darkness for $72 \mathrm{~h}$ at $28^{\circ} \mathrm{C}$. Yellow fluorescence was assessed using a fluorescence microscope (Olympus BH2) equipped with a $530 \mathrm{~nm}$ filter using exactly the same parameters for all the images captured (adjusted with the auto level function and colour replacement function in Adobephotoshop6 $\left.{ }^{\circledR}\right)$.

The positive control for the BiFC experiments was a stable pSPYNE 35S Arath;bZIP63 tobacco BY-2 line transiently transformed with pSPYCE 35S Arath;bZIP63 [31]. The negative control was a stable pSPYNE 35S Arath;bZIP63 tobacco BY-2 line transiently transformed with pSPYCE 35S Arath;WEE1. For the detection of callose on cell cross-walls, $20 \mu \mathrm{l}$ of BY-2 cells was mixed with $0.01 \%(\mathrm{w} / \mathrm{v})$ aniline blue (Gurr-BDH, UK) in a $3 \%(\mathrm{w} / \mathrm{v})$ solution of ANALAR sucrose (BDH,UK). Slides were left for $1 \mathrm{~h}$ before being examined using fluorescence microscopy (as detailed above).

\section{Site-Directed Mutagenesis}

Site-directed mutagenesis of Arath;WEE1 was performed using the QuickChange ${ }^{\circledR}$ Site-directed Mutagenesis kit (Stratagene) with primers FW: 5'- GTCGGCCTGCTGCTAGAGAATTACTGGAC-3' and RV: 5'- CTAGCAGCAGGCCGACGCTTCGGATC-3'. The PCR program was: 1 cycle of initial denaturation $\left(30 \mathrm{~s}\right.$ at $\left.95^{\circ} \mathrm{C}\right) ; 16$ cycles of denaturation $\left(30 \mathrm{~s}\right.$ at $95^{\circ} \mathrm{C}$; annealing $\left(1 \mathrm{~min}\right.$ at $\left.55^{\circ} \mathrm{C}\right)$; extension $(20$ $\min$ at $\left.68^{\circ} \mathrm{C}\right) ; 1$ cycle of final extension $\left(1 \mathrm{~h}\right.$ at $\left.68^{\circ} \mathrm{C}\right)$. PCR reactions were digested with $10 \mathrm{U} \mathrm{DpnI}$ for $1 \mathrm{~h}$ at $37^{\circ} \mathrm{C}$ and EtOH precipitated. The precipitated DNA was transformed into $E$. coli $\mathrm{DH} 5 \alpha$ cells and the S485A mutation was verified by sequencing of the original mutagenised plasmid. The Arath;WEE1 (S485A) coding sequence was re-cloned into an empty pBD-Gal4-cam vector via SalI/EcoRI restriction sites and into the empty pSPYCE 35S vector with SpeI/XhoI restriction sites as previously described.

\section{RESULTS}

\section{Arath; WEE1 Interacts with the Non-Epsilon Group of} 14-3-3s

Previous work established that some of the Non-Epsilon 14-3-3 proteins could rescue cell cycle defects in fission yeast mutants [28]. Hence, a targeted yeast two hybrid $(\mathrm{Y} 2 \mathrm{H})$ screen tested whether Arath;WEE1 interacted with the Non-Epsilon group of 14-3-3 proteins comprising:

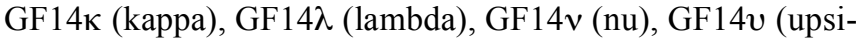
lon), GF14 $\varphi$ (phi), GF14 $\chi$ (chi), GF14 $\psi$ (psi) and GF14 $\omega$ (omega). Bait and target plasmids were co-transformed into yeast and transformants were detected if they grew on me- 
dium deficient in tryptophan and leucine. Transformants carrying the Arath;WEEl bait plasmid co-transformed with the Non-Epsilon 14-3-3 target plasmids grew on medium lacking histidine indicating transcriptional activation of the HIS3 reporter gene by an interaction between Arath;WEE1 and all of the Non-Epsilon 14-3-3 proteins (data not shown). In a second screen, the interaction was verified when the transformants carrying the Arath;WEE1 bait plasmid, cotransformed with the Non-Epsilon 14-3-3 target plasmids, activated transcription of the $L a c Z$ reporter gene in a nonquantitative assay (blue colouration of the yeast in the filter lift assay, Fig. 1).

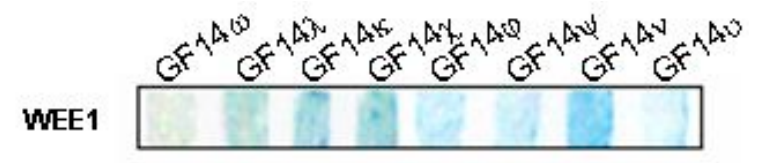

WEE1 only

Fig. (1). Arath; WEE1 interacts with Non Epsilon 14-3-3 proteins in vitro. Qualitative filter lift assay of yeast colonies coexpressing Arath:WEE1 and 14-3-3 proteins belonging to the NonEpsilon group as a control, yeast transformed with the Arath;WEE1 bait plasmid only was used showing that the Arath;WEE1 bait is not capable of auto-activating the $L a c Z$ reporter gene.

Interaction Between Arath;WEE1 and Arath;GF14 $\omega$ Verified by Co-Immunoprecipitation

Of three interacting Non-Epsilon 14-3-3 proteins, GF $14 \kappa, \lambda$ and $\omega$, only the latter was expressed more strongly in proliferative regions but less so in non-proliferative regions [29]. Hence, we focussed on the WEE1/GF14w interaction which was confirmed in vitro by coimmunoprecipitation using the pESC yeast epitope-tagging vector system (Stratagene). Arath;WEE1 was tagged with c-myc, while Arath;GF14 $\omega$ was FLAG tagged. Proteins were pulled down with the anti-FLAG antibody, and Western blots probed with FLAG and c-myc antibodies (Fig. 2A) revealed a band of $56 \mathrm{kDa}$ corresponding to the size of Arath;WEE1 in the Arath;WEE1 c-myc/Arath;GF14 $\omega$ FLAG eluate, indicating that Arath;WEE1 binds to Arath; GF14 $\omega$. The $56 \mathrm{kDa}$ band corresponding to the size of Arath;WEE1 was not detectable in the control coimmunoprecipitations (Arath;GF14 $\omega$ FLAG only or Arath;

A

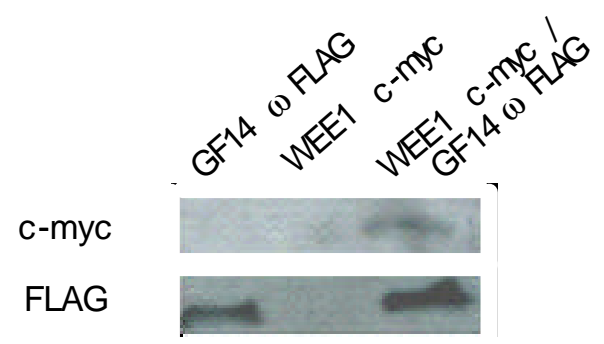

WEE1 c-myc only) and, a band of $29 \mathrm{kDa}$ corresponding to the size of Arath;GF14 $\omega$ was detected in all coimmunoprecipitation eluates except in the Arath;WEE1 cmyc control (Fig. 2A). Additionally, Arath;GF14 $\omega$ could bind to native Arath;WEE1. A total protein extract from Arabidopsis seedlings was mixed with protein extracted from the Arath;GF14 $\omega$ FLAG-producing yeast and when proteins were pulled down with anti FLAG antibody and Western blots were probed with an antibody raised against plant WEE1, a band of $56 \mathrm{kDa}$ corresponding to the size of Arath;WEE1 could be detected (Fig. 2B).

\section{In Vivo Interaction Between Arath;WEE1 and Arath;GF14 $\omega$ Shown by Bi-molecular Fluorescence Complementation (BiFC)}

The BiFC technique can analyze protein-protein interactions in a wide-range of plant tissues and cell types. So far it has been used in foliage by leaf infiltration techniques or in plant cell-derived protoplasts by transient transformation $[32,33]$. To our knowledge, this is the first report of a functional BiFC system in plant cell suspension culture. This has the advantage over the use of protoplasts in providing sub-cellular localization and, unlike the use of leaf infiltrations, does not require confocal microscopy. Analyses were performed by Agrobacterium-mediated transient transformation of stable tobacco BY-2 lines carrying one of the two split-YFP constructs in the pSPYNE vector. pSPYNE 35S Arath;bZIP63 and pSPYCE 35S Arath;bZIP63 were the positive controls and in BY2 interphase cells, yellow fluorescence was detected in the nucleus (Fig. 3A), in agreement with the use of these vectors in protoplasts (Walter et al., 2004). A stable tobacco BY-2 cell culture carrying pSPYNE 35S Arath;bZIP63 transformed transiently with pSPYCE $35 \mathrm{~S}$ Arath;WEE1 was used as the negative control and fluorescence could not be observed (Fig. 3B). Since 14-3-3 proteins can form dimers [34-36], a stable BY2 cell line carrying the pSPYNE 35S Arath;GF14 $\omega$ was transiently transformed with pSPYCE $35 \mathrm{~S}$ Arath;GF14 $\omega$. In contrast to the Arath:bZIP63, fluorescence was seen mainly in the nucleus but also in the wall between adjacent cells (Fig. 3C).

When a tobacco BY-2 cell culture carrying pSPYNE 35S Arath; $G F 14 \omega$ was transiently transformed with pSPYCE 35S Arath;WEE1, yellow fluorescence was detected in

\section{B}

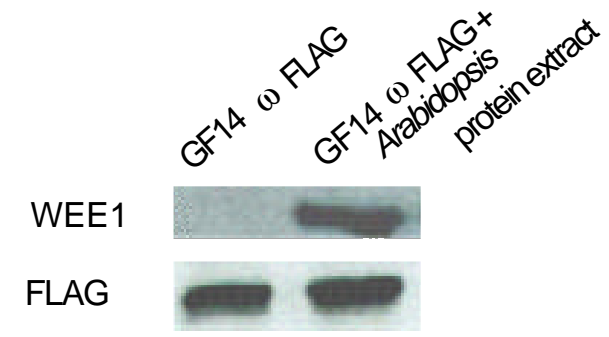

Fig. (2). Biochemical confirmation of the interaction in vitro. Co-immunoprecipitation analysis of Arath;WEE1 and Arath;GF14w (A) FLAG and c-myc antibody-probed western blots of FLAG immunoprecipitated proteins extracted from yeast transformants carrying pESC constructs: Arath;GF14 $\omega$ FLAG only, Arath;WEE1 c-myc only, Arath;WEE1 c-myc/ GF14 $\omega$ FLAG. (B) FLAG and WEE1 antibody-probed western blots of FLAG immunoprecipitated proteins extracted from yeast transformants carrying pESC constructs: Arath;GF14 $\omega$ FLAG only and Arath;GF14 $\omega$ FLAG mixed with $1 \mathrm{mg}$ of Arabidopsis protein extract. 
interphase cells in the nucleus (Fig. 4A) and in the periphery of the cytoplasm (Fig. 4B). However, yellow fluorescence was also prominent in the cell wall between shorter cells (Fig. 4C). We hypothesise that this latter signal is associated with the cell plate at cytokinesis. To test this, we used aniline blue as a marker of cytokinesis (Fig. 5); it stains callose at the newly forming cell plate [37]. There was a very low frequency $(0.03 \%)$ of such stained cross walls in comparable cells used for BiFC. Thus the very low frequency of both BIFC positive signal on cross walls between small cells (Fig. 4C) and stained cross walls by aniline blue, suggests an interaction between WEE1 and GF14 $\omega$ at cytokinesis.
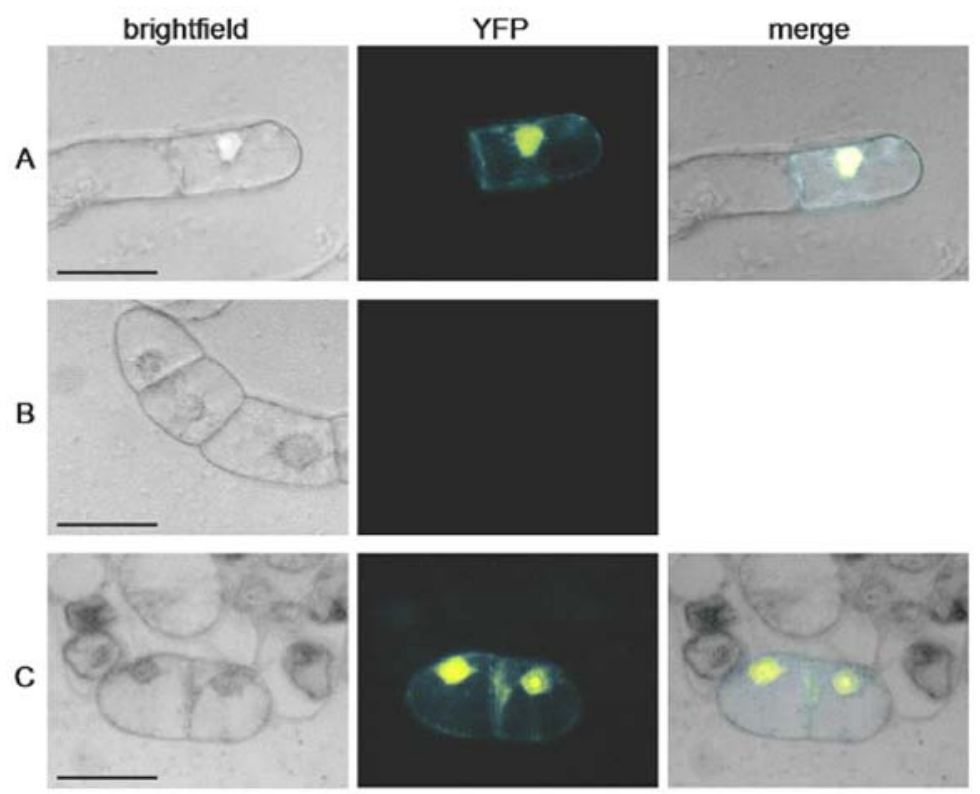

Fig. (3). Confirmation of SPYCE/SPYNE interaction in vivo. BiFC analysis of (A) the Arath;bZIP63/Arath;bZIP63 homodimerization (positive control), transient Agrobacterium-mediated transformation of the pSPYNE 35S Arath;bZIP63 BY-2 cell line with pSPYCE 35S Arath;bZIP63. Yellow fluorescence could be detected only in the nucleus. (B) Arath;bZIP63/Arath;WEE1 (negative control), transient Agrobacterium-mediated transformation of the pSPYNE 35S Arath;bZIP63 BY-2 cell line with pSPYCE 35S Arath;WEE1. Yellow fluorescence could not be detected. (C) homodimerisation of Arath;GF14 Arath;GF14 $\omega$ BY-2 cell line with pSPYCE 35S Arath;GF14 $\omega$. Yellow fluorescence was also detected in the nucleus and a small mid section of the cell wall formed between adjacent cells. YFP images were detected at an approximate frequency of 1:6000 Bar scale $=50 \mu \mathrm{m}$.
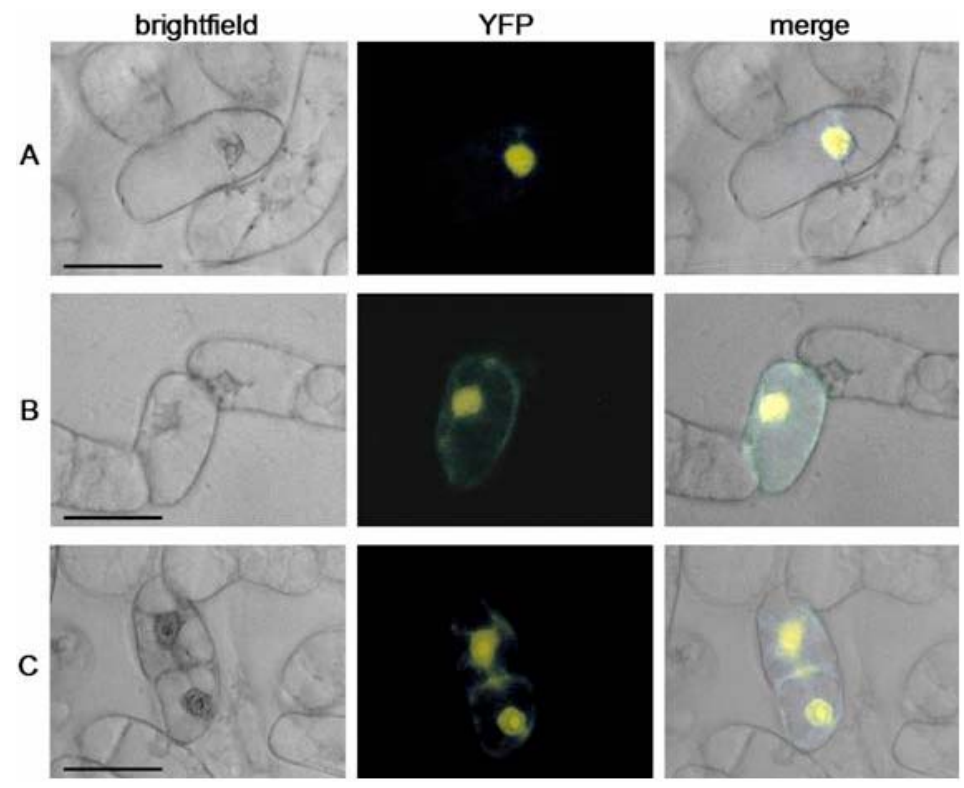

Fig. (4). Cytoplasmic and nuclear Interaction between WEE1 and GF14 $\omega$ in vivo. BiFC analysis of the Arath; WEE1/Arath;GF14 $\omega$ interaction by transient Agrobacterium-mediated transformation of the pSPYNE 35S Arath;GF14 $\omega$ BY-2 cell line with pSPYCE 35S Arath;WEE1. Yellow fluorescence could be detected (A) in the nucleus $(\mathbf{B})$, cell wall of interphase cells and $(\mathbf{C})$ in peripheral cytoplasm and in the wall between two cells. Note that the length of cells in $4 \mathrm{C}$ are approximately half that of the labelled cell in $4 \mathrm{~A}$ suggesting that the former were recently divided. YFP images were detected at an approximate frequency of 1 in 6000 . Bar scale $=50 \mu \mathrm{m}$. 
Mutation of the Arath;WEE1 14-3-3 Binding Site Perturbs Interaction Between Arath;WEE1 and Arath; GF14 $\omega$

Using motif scanning software (http://scansite.mit.edu/ motifscan_seq.phtml), five putative mode I (RSXpSXP) 143-3 binding motifs were identified in the protein sequence of Arath;WEE1 (Fig. 6A) allowing for low stringency matching. The putative 14-3-3 binding site located at S485 in the C-terminal of Arath;WEE1, displays high similarity to the 14-3-3 binding sites located at S549/S558 in mouse and human WEE1, respectively but less so to the corresponding S559 site in Xenopus (Fig. 6B). Therefore, S485 in the Cterminal of Arath;WEE1 was considered to be the most likely binding site for Arath;GF14w. An Arath;wee1 (S485A) mutant was constructed by site-directed mutagenesis and $\mathrm{Y} 2 \mathrm{H}$ used to test whether the abolition of this site in Arath;WEE1 affected the interaction with Arath;GF14 $\omega$. Growth was not observed when the weel(S485A)/Arath; GF14W transformant was grown on medium deficient in histidine indicating that the S485A mutation of Arath;WEE1 does indeed abolish Arath;GF14 $\omega$ binding. This was confirmed by a $L a c Z$ reporter gene filter lift assay and a quantitative liquid ONPG assay (Figs. 7A and B). To test if the interaction was abolished in vivo, the tobacco BY-2 cell line carrying pSPYNE 35S Arath;GF14 $\omega$ was transiently transformed with pSPYCE 35S Arath;WEE1 (S485A). Fluorescence was still detected in the nucleus of interphase cells (Fig. 8) but no longer in the cell wall between cells as judged by a scan of $\geq 6,000$ cells (Fig. 8) suggesting that the Arath;WEE1 (S485A) mutation perturbs the binding with Arath;GF14 $\omega$ by restricting the localization of Arath;WEE1 to the nucleus.
A

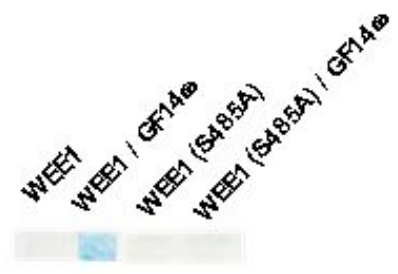

B

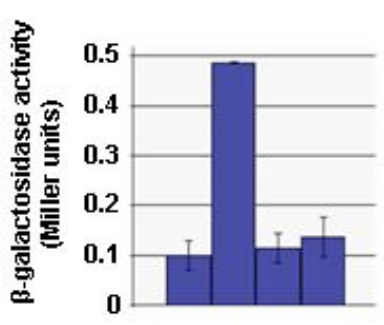

Fig. (7). Mutated WEE1 does not interact with GF14 $\omega$ in vitro. LacZ reporter gene screening: (A) Filter lift assay, from left: Arath;WEE1 only, Arath;WEE1/Arath;GF14 $\omega$, Arath;WEE1 (S485A) only and Arath;WEE1 (S485A)/Arath;GF14 $\omega$. (B) Quantitative ONPG assay, from left Arath;WEE1 only, Arath;WEE1/

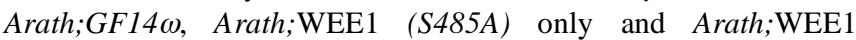

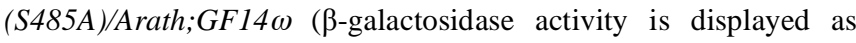
mean Miller units $\pm \mathrm{SE}, \mathrm{n}=3$ ).

\section{DISCUSSION}

\section{Arath; WEE1/GF14 $\omega$ Interaction}

A targeted $\mathrm{Y} 2 \mathrm{H}$ screen showed that Arath;WEE1 interacts with all the Arabidopsis Non-Epsilon 14-3-3 isoforms which is consistent with this protein family displaying high sequence conservation $(70-90 \%)$ both at the nucleotide and protein level [44]. Moreover, four of the Non-Epsilon 14-3-3

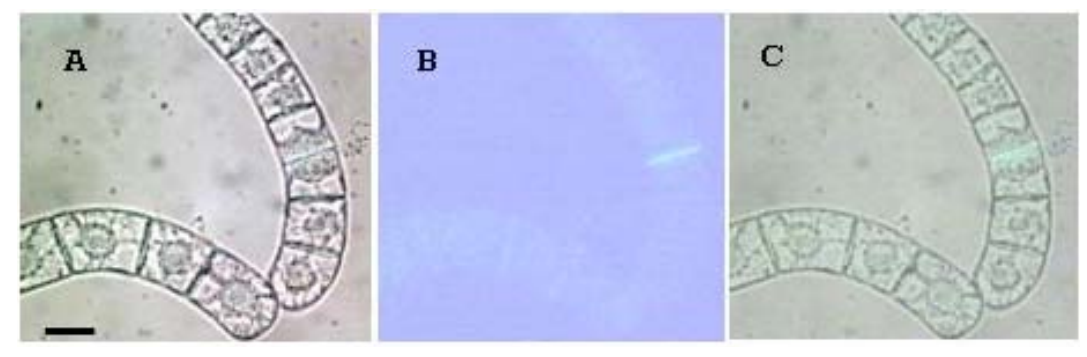

Fig. (5). Cytokinesis. BY-2 cells stained with $0.02 \%(w / v)$ aniline blue (A) bright field illumination (B) fluorescence at $420 \mathrm{~nm}$ excitation filter (adjusted with auto levels (Adobephotoshop6®)) (C) merged image of (A) on greyscale and the primary fluorescent image of (B). Bar scale $=50 \mu \mathrm{m}$.
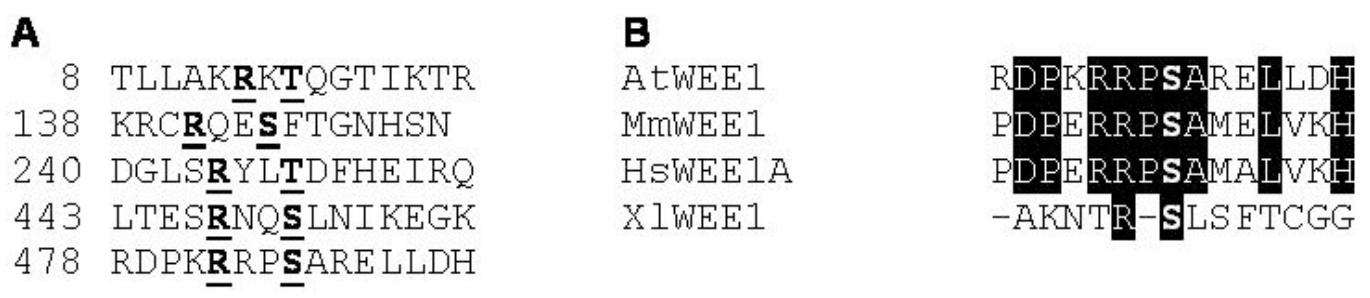

Fig. (6). Mutation of Arath;WEE1. (A) The five putative 14-3-3 binding sites identified in the Arath;WEE1 protein sequence using a motif scanning software (http://scansite.mit.edu/motifscan_seq.phtml) and the predicted phosphorylation sites (numbers indicate amino acid residue in the Arath;WEE1 protein sequence). (B) Line up of the Serine 485 binding site for WEE1 in Arabidopsis thaliana (At), with S549 in Mus musculus (Mm), S558 in Homo sapiens (Hs) and S559 in Xenopus laevis (Xl). 


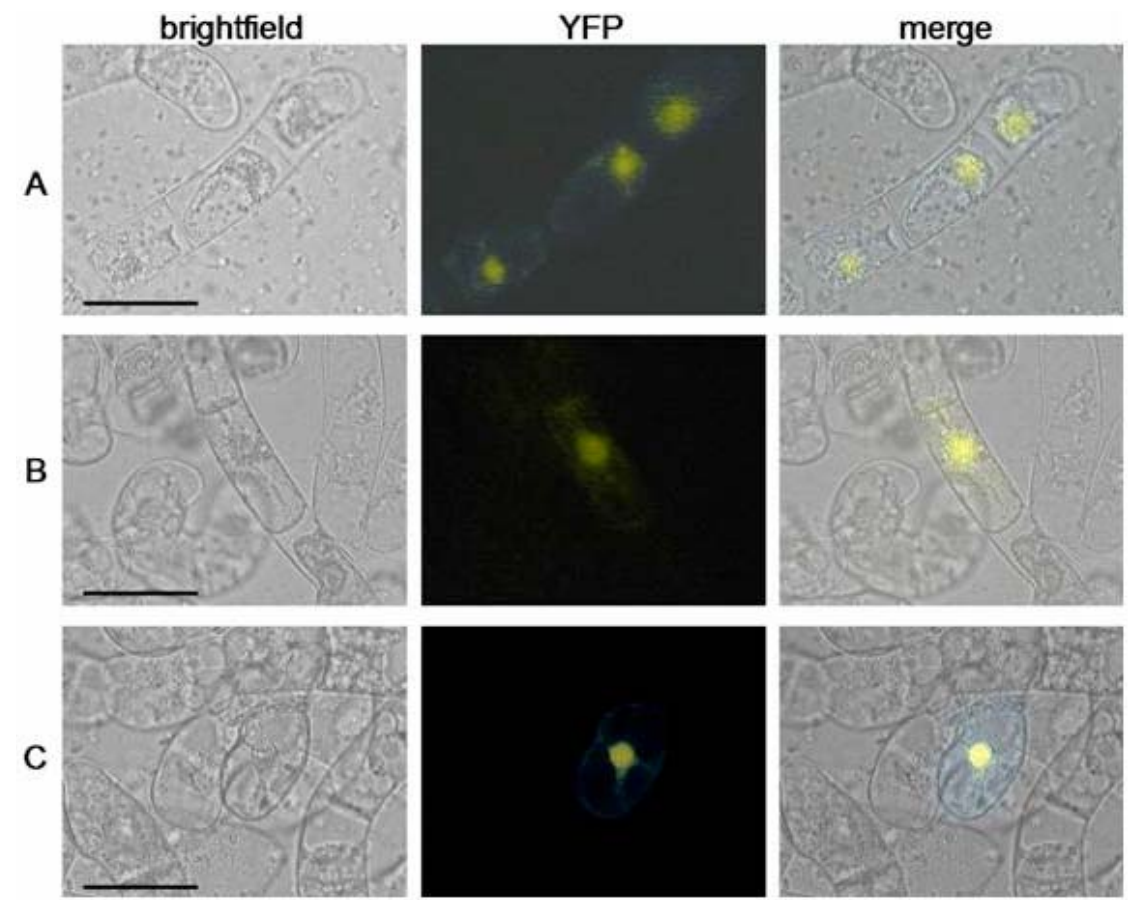

Fig. (8). Mutated WEE1 does not interact with GF14 $\omega$ at the cell wall. BiFC analysis of the Arath;WEE1 (S485A)/Arath;GF14 $\omega$ interaction by transient Agrobacterium-mediated transformation of the pSPYNE 35S Arath;GF14 $\omega$ BY-2 cell line with pSPYCE 35S Arath;WEE1 (S485A). After $72 \mathrm{~h}$ yellow fluorescence could only be detected in the nuclei (A, B and C). YFP images were detected at an approximate frequency of 1:6000. Bar scale $=50 \mu \mathrm{m}$.

proteins tested here $(\mathrm{GF} 14 \varphi, \chi, v$ and $\omega)$ could rescue the rad $24^{-}$checkpoint mutant of $S$. pombe [28]. The work then focussed on the Non-Epsilon GF14w because like Arath;WEE1 [7], it is relatively more highly expressed at the transcript level in proliferative regions of Arabidopis plants [29] including young roots (data not shown), although unlike Arath;WEE1 it is not cell-cycle regulated [38], (and data from Atgene Express).

The specificity of the interaction between Arath;WEE1 and Arath;GF14 $\omega$ was confirmed in vitro by coimmunoprecipitation of recombinant proteins. Five mode I 14-3-3 protein binding sites were identified within the Arath;WEE1 protein sequence of which one, at S485, showed high similarity to 14-3-3 binding sites reported for other eukaryotes [19-21]. The arath;wee1 (S485A) mutant protein was incapable of interacting with Arath;GF14 $\omega$ in a $\mathrm{Y} 2 \mathrm{H}$ screen, suggesting strongly that S485 is the binding site for Arath;GF14 1 . A quantitative ONPG assay was performed, which further indicated that the interaction was indeed abolished by the S485A mutation.

\section{Spatial Sub-cellular Localisation}

BiFC, besides confirming the Arath;WEE1/GF14 $\omega$ interaction, indicated that the interaction was located predominantly in the nucleus of interphase cells but additionally in cytoplasmic strands and, we suggest, the wall that forms at cytokinesis. Moreover the frequency of aniline blue-stained cross walls at cytokinesis and $\mathrm{BiFC}$ positive cells suggest that the interaction may be at the cell plate. This suggested distribution would be similar to previous work in Arabidopsis hypocotyls where a GF14 $\omega$ C-terminal:GFP fusion localised to the nucleus, phragmoplast and cytoplasmic periphery of cells undergoing cytokinesis [39]. However, whilst our localisation of Arath;WEE1/GF14 $\omega$ for interphase cells was nuclear, most of their GF14 $\omega:$ GFP localisations in interphase cells were cytoplasmic. This differential localisation of GF14 $\omega$ may well be because BY-2 interphase cells are predominantly proliferative whereas in Arabidopsis hypocotyls, interphase cells are mostly differentiated. GF14 $\omega:$ GFP subcellular localisation in Arabidopsis trichomes and guard cells indicated a uniform distribution in the nucleus as well as a spread to components of the cytoskeleton throughout the cytoplasm and cell wall [40] providing a closer match to the GF14 $\omega$ homodimer localisation reported here although our cell wall localisations are much weaker than theirs.

When the arath;weel (S485A) mutant protein was tested against GF14 1 , yellow fluorescence was restricted to the nucleus; it was not detected either in walls between cells or the cytoplasmic periphery of the cell. This suggests that the S485A mutation in the Arath;WEE1 affects the binding in plant cells such that the interaction with Arath;GF14 $\omega$ can no longer occur outside of the nucleus. However the interaction was not completely abolished as might have been expected from the $\mathrm{Y} 2 \mathrm{H}$ results. This suggests that the interaction in plant cells may be more complex. When all phosphorylation sites within the three 14-3-3 binding motifs of $S$. pombe Cdc25 were mutated, this reduced the affinity of the binding between 14-3-3 and Cdc25 but did not abolish it [22]. Thus the interaction between Arath;GF14 $\omega$ and arath;wee1 (S485A) may likewise be reduced in affinity and only partially abolished due to the presence of other factors such as other interacting proteins. The BiFC fluorescence cannot be treated as quantitative and hence, a reduction in affinity of the interaction throughout the cell cannot be 
excluded. In Xenopus, 14-3-3e binding keeps WEE1 evenly distributed in the nucleus during interphase [20] and is a nuclear protectant from mitogenic cdk-like activity [15]. In human mitotic cells, WEE1 is similarly sequestered in the cytoplasm when an $\mathrm{AKt} /$ protein kinase B, phosphorylates WEE1 creating a binding site for 14-3-30 [41]. Our observations support a distinctive and differential subcellular distribution of GF14 $\omega$ when bound to its client, Arath;WEE1.

HsWEE1 started to re-associate with the nucleus at telophase and in particular was located on the cleavage furrow during cytokinesis [9]. At that time, the authors commented on a cytoplasmic localisation of WEE1 with unknown cytoplasmic factors which might well have been a tethering to a 14-3-3-protein. Arath;WEE1/GF14 $\omega$ localisation to the cell plate would fit well with these observations and is intriguing because Arath;WEE1 scored multiple hits with Arath;CDC48A, in a Y2H assay (A. Lentz Grønlund, unpublished data), a protein known to be involved in vesicle formation during plant cytokinesis [42].

In conclusion, our data are consistent with an interaction between Arath;WEE1 and Arath;GF14 $\omega$ both in vitro and in vivo. However, binding between WEE1 and GF14 in the cytoplasm is likely to be the key interaction and would fit with a requirement to disperse WEE1 into the cytoplasm prior to the onset of mitosis.

\section{ACKNOWLEDGEMENTS}

We thank Cardiff University, University of Worcester and BBSRC for funding this work and Dr J Kudla (Münster Univ., GE) for kindly supplying us with the BiFC vectors.

\section{REFERENCES}

[1] Morgan DO. Cyclin-dependent kinases: engines, clocks, and microprocessors. Annu Rev Cell Dev Biol 2007; 13: 61-91.

[2] Norbury C, Nurse P. Animal cell cycles and their control. Annu Rev Biochem 1992; 61: 41-70.

[3] O'Farrell PH. Triggering the all-or-nothing switch into mitosis. Trends Cell Biol 2001; 11: 12-19.

[4] Russell P, Nurse P. Negative regulation of mitosis by wee1+, a gene encoding a protein kinase homolog. Cell 1987; 49: 59-67.

[5] McGowan CH, Russell P. Cell cycle regulation of human WEE1. EMBO J 1995; 14: 66-75.

[6] Sun Y, Dilkes BP, Zhang C, et al. Characterization of maize (Zea mays L.) Weel and its activity in developing endosperm. Proc Natl Acad Sci USA 1999; 96: 80-85.

[7] Sorrell DA, Marchbank A, McMahon K, Dickinson JR, Rogers HJ, Francis D. A WEE1 homologue from Arabidopsis thaliana. Planta 2002; 215: 18-22.

[8] Gonzalez N, Hernould M, Delmas F, et al. Molecular characterization of a WEE1 gene homologue in tomato (Lycopersicon esculentum Mill.). Plant Mol Biol 2004; 56: 49-61.

[9] Baldin V, Ducommun B. Subcellular localisation of human wee1 kinase is regulated during the cell cycle. J Cell Sci 1995; 108: 2532.

[10] Elledge SJ. Cell cycle checkpoints: preventing an identity crisis. Science 1996; 274: 64-72.

[11] Weinert T. DNA damage checkpoints update: getting molecular. Curr Opin Genet Dev 1998; 8: 85-93.

[12] Abraham J, Kelly J, Thibault P, Benchimol S. Post-translational modification of $\mathrm{p} 53$ protein in response to ionizing radiation analyzed by mass spectrometry. J Mol Biol 2000; 295: 53-64.

[13] Branzei D, Foiani M. Regulation of DNA repair througout the cell cycle. Nat Rev Mol Cell Biol 2008; 9: 97-08.

[14] Rhind N, Russell P. Chk1 and Cds1: linchpins of the DNA damage and replication checkpoint pathways. J Cell Sci 2000; 113: 89-96.

[15] Perry JA, Kornbluth S. Cdc25 and Wee1: antagonistic opposites. Cell Div 2007; doi:10.1186/1747-1028-2-2.
[16] Forbes KC, Humphrey T, Enoch T. Suppressors of cdc25p overexpression identify two pathways that influence the G2/M checkpoint in fission yeast. Genetics 1998; 150: 61-75.

[17] Kumagai A, Dunphy WG. Binding of 14-3-3 proteins and nuclear export control the intracellular localization of the mitotic inducer Cdc25. Genes Dev 1999; 13: 67-72.

[18] Yang J, Winkler K, Yoshida M, Kornbluth S. Maintenance of G2 arrest in the Xenopus oocyte: a role for 14-3-3-mediated inhibition of Cdc25 nuclear import. EMBO J 1999; 18: 74-83.

[19] Wang Y, Jacobs C, Hook KE, Duan H, Booher RN, Sun Y. Binding of 14-3-3beta to the carboxyl terminus of Wee1 increases Wee 1 stability, kinase activity, and G2-M cell population. Cell Growth Differ 2000; 11: 11-19.

[20] Lee J, Kumagai A, Dunphy WG. Positive regulation of Wee1 by Chk1 and 14-3-3 proteins. Mol Biol Cell 2001; 12: 51-63.

[21] Rothblum-Oviatt CJ, Ryan CE, Piwnica-Worms H. 14-3-3 binding regulates catalytic activity of human Weel kinase. Cell Growth Differ 2001; 12: 81-89.

[22] Zeng Y, Piwnica-Worms H. DNA damage and replication checkpoints in fission yeast require nuclear exclusion of the Cdc25 phosphatase via 14-3-3 binding. Mol Cell Biol 1999; 19: 10-19.

[23] Graves PR, Lovly CM, Uy GL, Piwnica-Worms H. Localization of human $\mathrm{Cdc} 25 \mathrm{C}$ is regulated both by nuclear export and 14-3-3 protein binding. Oncogene 2001; 20: 39-51.

[24] Yaffe MB, Rittinger K, Volinia S, et al. The structural basis for 143-3:phosphopeptide binding specificity. Cell 1997; 91: 61-71.

[25] Rittinger K, Budman J, Xu J, et al. Structural analysis of 14-3-3 phosphopeptide complexes identifies a dual role for the nuclear export signal of 14-3-3 in ligand binding. Mol Cell 1999; 4: 53-66.

[26] DeLille JM, Sehnke PC, Ferl RJ. The Arabidopsis 14-3-3 family of signaling regulators. Plant Physiol 2001; 126: 35-8.

[27] Rosenquist M, Sehnke P, Ferl RJ, Sommarin M, Larsson C. Evolution of the 14-3-3 protein family: does the large number of isoforms in multicellular organisms reflect functional specificity? J Mol Evol 2000; 51: 46-58.

[28] Kuromori T, Yamamoto M. Members of the Arabidopsis 14-3-3 gene family trans-complement two types of defects in fission yeast. Plant Sci 2000; 158: 55-61.

[29] Sorrell DA, Marchbank AM, Chrimes DA, et al. The Arabidopsis 14-3-3 protein, GF14omega, binds to the Schizosaccharomyces pombe Cdc25 phosphatase and rescues checkpoint defects in the rad24- mutant. Planta 2003; 218: 50-57.

[30] De Schutter K, Joubes J, Cools T, et al. Arabidopsis WEE1 kinase controls cell cycle arrest in response to activation of the DNA integrity checkpoint. Plant Cell 2007; 19: 11-25.

[31] Walter M, Chaban C, Schutze K, et al. Visualization of protein interactions in living plant cells using bimolecular fluorescence complementation. Plant J 2004; 40: 28-38.

[32] Bhat RA, Lahaye T, Panstruga R. The visible touch: in planta visualization of protein-protein interactions by fluorophore-based methods. Plant Methods 2006; 2: 12.

[33] Ohad N, Shichrur K, Yalovsky S. The analysis of protein-protein interactions in plants by bimolecular fluorescence complementation. Plant Physiol 2007; 145: 90-99.

[34] Muslin AJ, Tanner JW, Allen PM, Shaw AS. Interaction of 14-3-3 with signaling proteins is mediated by the recognition of phosphoserine. Cell 1996; 84: 89-97.

[35] Muslin AJ, Xing H. 14-3-3 proteins: regulation of subcellular localization by molecular interference. Cell Signal 2000; 12: 3-9.

[36] Chaudhri M, Scarabel M, Aitken A. Mammalian and yeast 14-3-3 isoforms form distinct patterns of dimers in vivo. Biochem Biophys Res Commun 2003; 300: 79-85.

[37] Buschmann H, Chan J, Sanchez-Pulido L, Andrade-Navaro MA, Doonan JH, Lloyd CW. Microtubule associated AIR-9 recognizes the cortical division site at pre-prophase and cell plate insertion. Curr Bio 2006; 16: 38-43.

[38] Menges M, De Jager SM, Gruisssem W, Murray JAH. Global analysis of the core cell cycle regulators of Arabidopis identifies novel genes, reveals multiple and highly specific profiles of expression and provides a coherent model for plant cell cycle control. Plant J 2005; 41: 46-66.

[39] Cutler SR, Ehrhardt DW, Griffiths JS, Somerville CR. Random GFP::cDNA fusions enable visualization of subcellular structures in cells of Arabidopsis at a high frequency. Proc Natl Acad Sci USA 2000; 97: 18-23. 
[40] Paul A-L, Sehnke PC, Ferl RJ. Isoform-specific subcelluar localisation among 14-3-3 proteins in Arabidopsis seems to be driven by client interactions. Mol Biol Cell 2005; 16: 35-43.

[41] Katayama K, Fujita N, Tsuruo T. Aky/protein kinsase B-dependent phosphorylation and inactivation of WEE1Hu promote cell cycle progression at G2/M. Mol Cell Biol 2005; 25: 25-37.

[42] Rancour DM, Dickey CE, Park S, Bednarek SY. Characterization of AtCDC48. Evidence for multiple membrane fusion mechanisms at the plane of cell division in plants. Plant Physiol 2002; 130: 4153.

[43] An GH. High efficiency transformation of cultured tobacco cells Plant Physiol 1985; 79: 568-70.

[44] Wu K, Rooney MF, Ferl RJ. The Arabidopsis 14-3-3 multigene family. Plant Physiol 1997; 114: 1421-31.

(C) Grønlund et al.; Licensee Bentham Open.

This is an open access article licensed under the terms of the Creative Commons Attribution Non-Commercial License (http://creativecommons.org/licenses/bync/3.0/) which permits unrestricted, non-commercial use, distribution and reproduction in any medium, provided the work is properly cited. 